\title{
Anne-Chantal Hardy, Travailler à guérir : sociologie de l'objet du travail médical
}

Rennes, Presses de l'École des hautes études en santé publique, coll. « Recherche, santé, social », 2013

\section{Sylvie Célérier}

\section{(2) OpenEdition}

\section{Journals}

Édition électronique

URL : http://journals.openedition.org/travailemploi/6277

DOI : $10.4000 /$ travailemploi.6277

ISSN : 1775-416X

Éditeur

DARES - Ministère du Travail

Édition imprimée

Date de publication : 15 mars 2014

Pagination : 140-142

ISSN : 0224-4365

Référence électronique

Sylvie Célérier, « Anne-Chantal Hardy, Travailler à guérir : sociologie de l'objet du travail médical », Travail et Emploi [En ligne], 137 | janvier-mars 2014, mis en ligne le 01 janvier 2014, consulté le 22 septembre 2020. URL : http://journals.openedition.org/travailemploi/6277 ; DOI : https://doi.org/10.4000/ travailemploi.6277

Ce document a été généré automatiquement le 22 septembre 2020.

(C) Direction de l'animation de la recherche, des études et des statistiques (Dares) 


\section{Anne-Chantal Hardy, Travailler à guérir : sociologie de l'objet du travail médical}

Rennes, Presses de l'École des hautes études en santé publique, coll. « Recherche, santé, social », 2013

\section{Sylvie Célérier}

\section{RÉFÉRENCE}

Anne-Chantal Hardy, Travailler à guérir : sociologie de l'objet du travail médical, Rennes, Presses de l'École des hautes études en santé publique, coll. « Recherche, santé, social », 2013, $301 \mathrm{p}$.

1 Le livre d'Anne-Chantal Hardy nous invite à la réflexion sur des objets dont on pensait tout connaître. À la question : "que font les médecins? ", chacun répond en effet que ces professionnels guérissent ou tentent de guérir les personnes venues les consulter sur les problèmes de santé qu'elles rencontrent. Si l'on se méfie de cette expérience immédiate, les sciences humaines offrent quelques rayonnages de références essentielles où la profession médicale miroite de multiples facettes que les chercheurs ont minutieusement explorées au cours des cinquante dernières années. L'autonomie professionnelle des médecins, cette capacité à s'auto-instituer et à contrôler sa propre activité, est un des ressorts de ce large intérêt qui explique aussi le statut d'archétype régulièrement conféré à la profession médicale par les analystes des professions. Bref, on sait bien des choses sur les médecins, leurs pratiques ainsi que sur leurs patients que nous sommes tous à un moment ou l'autre de notre vie.

2 Anne-Chantal Hardy parvient cependant à rafraîchir notre regard sur cet univers si émotionnellement et conceptuellement peuplé. Elle y emploie un riche matériau empirique accumulé au cours de son expérience de recherche sur les mondes de la médecine, sur la formation professionnelle, les métiers de la coiffure ou la 
professionnalisation de la marine nationale. La sociologie que défend l'auteure ne s'enferme pas en effet dans les frontières du social et, ce qui s'observe ici, doit profiter de ce qui a été compris ailleurs. Au-delà de cette solide assise empirique, Anne-Chantal Hardy emprunte une voie originale pour entrer dans la profession. Elle y combine trois grands principes, rarement rassemblés, qui innervent l'ensemble de l'ouvrage en lui donnant la forme d'un développement plutôt qu'une distribution rigide entre les quatre parties qui le composent. Parties qui se font donc mutuellement écho.

3 Premier principe : une profession ne se comprend vraiment qu'en élucidant l'activité concrète de ses membres et ce qui fait sens pour eux. Ce principe, Anne-Chantal Hardy le traduit en une interrogation sur l'objet du travail médical qu'elle place au cœur de son ouvrage, dans une troisième partie qu'il faut comprendre comme un nœud dans le raisonnement. En amont, les deux premières parties ont exploré ce qui rend socialement possible, nécessaire et reproductible cet objet de travail et, en aval, la quatrième partie suit l'impact de cet objet pour ceux auxquels il s'applique, c'est-à-dire les patients.

4 La formation de l'objet médical (parties 1 et 2) est renvoyée à quelques moments clefs de l'institutionnalisation et de la transformation du groupe professionnel au cours de la première moitié $\mathrm{du} \mathrm{xx}^{\mathrm{e}}$ siècle en France. Chaque fois, l'auteure y découvre une complexité de relations et d'enjeux qui dépasse la lutte pour la reconnaissance et pour l'autonomie que les analystes des professions privilégient dans leur compréhension de la dynamique des groupes qu'ils étudient. Il faut, nous dit Anne-Chantal Hardy, élargir ce cadre et y intégrer la variété et la complexité des relations que la profession entretient avec les acteurs institutionnels qui participent de sa (re)production sociale. Elle décrit ainsi des rapports de délégation ou d'allégeance aux pouvoirs publics avec lesquels les segments dominants de la profession collaborent pour maintenir leur position et orienter le cours et le sens de l'activité médicale. La réforme de l'internat de médecine en 1982 qui bouleverse les règles de sélection et d'affectation aux différentes spécialités en donne un exemple très convaincant. L'auteure pointe également les rapports de contrôle, voire de contrainte, qu'exerce l'assureur public, qui structurent l'activité quotidienne des médecins et fixent leurs rémunérations. Rapports de dépendance, pourrait-on ajouter, à la recherche biomédicale, domaine des laboratoires, qui échappe à la médecine praticienne, mais qui en oriente toutes les pratiques. AnneChantal Hardy finit de nous convaincre de la (re)production sociale de la médecine en rappelant que son renouvellement enrôle aujourd'hui de plus en plus de femmes qui la transforment de l'intérieur par les valeurs qu'elles promeuvent, bien différentes de celles qui animaient les générations antérieures dominées par les hommes, notamment par leur moindre attrait pour l'exercice libéral.

5 L'activité des médecins est donc une activité déléguée, orientée, prescrite par des intérêts variés qui échappent aux professionnels et auxquels leur profession s'ajuste constamment, voire se soumet. Cette activité est ainsi pluridimensionnelle et déborde le cadre ordinaire de la rencontre des professionnels et de leurs patients que les médecins aiment à penser sur le mode du "colloque singulier" auquel ils sont chèrement attachés. Ce « colloque » est infiniment plus socialisé qu'il n'y paraît. Dans la quiétude du cabinet, quantité d'acteurs ou d'intérêts interviennent qui, pour être imperceptibles dans l'expérience immédiate, n'en sont pas moins agissants. Ce qui prévaut et guide l'action de ces professionnels ne se décide donc pas (seulement) à hauteur de leur compétence ou de leur probité - que l'auteure ne met nullement en 
cause -, mais dans les mouvements complexes des logiques économiques, sociales et politiques qui traversent et remodèlent constamment leur profession. L'objet du travail médical perd donc de son évidence, devient instable, mouvant, et Anne-Chantal Hardy nous invite à en poursuivre l'examen du point de vue de la guérison, objectif affiché de la profession et espoir des profanes qui recourent aux services qu'elle propose.

6 L'exploration de ce versant de l'activité médicale conduit à des constats troublants. L'objectif de guérison qui lie les deux parties en présence se traduit dans les faits par une mise à mal - «pour le bien des malades »- du corps sur lequel le professionnel agit. Les étudiants en font l'amère expérience à l'occasion du stage de deuxième année qui organise souvent leurs premiers contacts avec les corps souffrants. Cette violence des soins est patente dans les traitements des cancers dont Anne-Chantal Hardy saisit les enjeux dans le face-à-face des oncologues et des parents d'enfants atteints ; parents, plus ou moins convaincus par la nécessité de la dureté des protocoles et parents différemment traités, eux aussi, selon les dispositions sociales que les médecins croient détecter en eux. Les hiérarchies du social restent ici particulièrement actives et dessinent des itinéraires de soin bien différents selon les familles. Au fil de la réflexion, la notion de guérison se fait, elle aussi, plus incertaine jusqu'à déboucher sur une inquiétante béance entre ce que les professionnels tiennent pour guérison et ce que les malades expérimentent dans leur propre corps. Déclarés "guéris ", ils sont aussi, souvent, définitivement « mutilés ».

7 L'auteure fait alors surgir deux interrogations majeures où s'entend l'influence des travaux sur la norme et la normalité de Georges Canguilhem et de Michel Foucault son élève. Elle souligne d'abord combien les « actes » de soin restent à la seule discrétion des experts qui en conservent jalousement (et obscurément) le contrôle, éthiquement abrités par une finalité de guérison dont on ne sait finalement pas grand-chose. L'auteure montre par ailleurs la puissance performative du décret de guérison que profèrent ces experts : être "guéri » ou «en rémission » équivaut en effet à se voir déclarer actif, disponible pour la production, quelle que soit l'ampleur des séquelles ou des empreintes que les « traitements » ont laissées sur les corps. Être « guéri » suppose encore que les obligations, morales aussi bien qu'économiques, de la communauté sont levées. L'opacité qui entoure les pratiques de guérison participe ainsi du partage du normal et du pathologique, partage décisif pour le malade et pour l'ensemble des membres de la communauté ; soit une forme renouvelée de biopouvoir, plus diffuse que par le passé, mais non moins contraignante.

8 Deuxième principe : les médecins sont des travailleurs (à peu près) comme les autres. Pour comprendre leurs situations de travail, Anne-Chantal Hardy engage une approche classique de sociologie du travail écartant l'hypothèse fragile d'une irréductible spécificité de ces professionnels sur laquelle débutent la plupart des travaux sur les professions. Elle s'intéresse donc à la formation des médecins, leur sélection et, plus largement, aux conditions de reproduction de leur groupe. Elle en obtient plusieurs résultats intéressants. Elle note d'abord le paradoxe d'un mode de recrutement proche de celui des grands corps de l'État, mais qui, pour l'essentiel, alimente des emplois indépendants. On voit aussi la constance des sélections sociales qui privilégient les enfants des catégories supérieures et des familles de médecins; inégalités qui persistent dans le renouvellement plus féminin de la profession.

9 L'intérêt pour les modalités de recrutement amène l'auteure à s'interroger sur le rôle qu'y prend l'université qui paraît, là encore, bien paradoxal. L'institution détient le 
monopole des diplômes, mais semble ne rien transmettre à ses étudiants, particulièrement lors de leurs deux concours, quand ils auraient le plus grand besoin de cette transmission. Symétriquement, elle réussit pleinement à leur apprendre à se passer d'elle et à composer par eux-mêmes les ressources qui leur sont nécessaires ; soit un apprentissage de l'autonomie forcé et coûteux pour les individus. De la formation initiale des médecins, on retient aussi la singularité de leur processus de socialisation qui enchaîne brutalement des phases de fragmentation intense du groupe par le jeu agonistique des rivalités induit par les concours et des phases de rassemblement tout aussi intenses pour ceux qui ont franchi les épreuves.

Considérer la médecine comme un espace de travail ordinaire oblige enfin à interroger la nature de sa contribution au processus productif. L'auteure lie cette contribution à la naissance et à l'extension des assurances sociales après la seconde guerre mondiale qui changent radicalement les conditions d'exercice de la médecine en France. Le nombre de médecins explose à cette occasion et les assurances prennent une part décisive dans leur rémunération. Qu'ils soient ou non favorables à cette évolution - Anne-Chantal Hardy rappelle en effet la vivacité des débats qui opposèrent les tenants d'une médecine libérale et ceux qui défendaient une médecine plus sociale -, tous ont pris une part active dans la production et le contrôle des corps de travailleurs que réclamait (et réclame encore) le système productif. Anne-Chantal Hardy reprend ici la puissante intuition de Talcott Parsons ${ }^{1}$ quant au rôle de la médecine dans le contrôle de la déviance que constitue la maladie. Les médecins du travail ne sont donc pas seuls engagés dans les rapports entre la santé et le travail, la médecine, au sens large, y contribue tout autant, même si son activité se tient apparemment loin des institutions de travail.

11 Dernier principe que propose l'auteure : les phénomènes ne sont jamais réductibles à une dimension unique et ils doivent leur dynamisme aux multiples traits qui constamment les traversent. S'attachant à la médecine, Anne-Chantal Hardy fait effort pour en dérouler les composantes, suivant chacune dans sa profondeur historique, sous des angles variés et depuis d'autres champs disciplinaires que la sociologie, notamment le droit. D'où son goût pour les paradoxes et les contradictions qui sont signe, pour elle, que quelque chose de décisif se tient en coulisse de la scène qu'elle observe qu'il faut impérativement saisir pour en restituer le sens. Ces paradoxes, contradictions et autres discordances contribuent à raviver ainsi notre curiosité sur des objets que les théories ont parfois excessivement figés.

12 Certains regretteront peut-être que cette méthode ne livre ni synthèse définitive ni nouveau schéma explicatif de la profession médicale. Les traces encore perceptibles de l'exercice académique d'habilitation à diriger des recherches qui est à l'origine de l'ouvrage participent peut-être aussi d'un léger désarroi du lecteur devant la multiplicité des fils tirés et entrecroisés dans l'ouvrage. On ne retient pour notre part qu'un étonnement devant l'absence de deux références qu'on pensait incontournables sur le sujet traité. Anne-Chantal Hardy reconnaît à plusieurs reprises sa dette envers Eliot Friedson et son remarquable travail sur la profession médicale ${ }^{2}$, mais elle ne cite ni le travail classique d'Haroun Jamous et Bertrand Peloille sur la reproduction des élites lors de la formation des médecins ${ }^{3}$ ni, surtout, les travaux d'Andrew Abbott ${ }^{4}$, hors une rapide citation en conclusion.

13 Or, ce dernier auteur - aujourd'hui largement mobilisé dans les travaux sur les professions - accorde lui aussi une place prépondérante aux activités concrètes des 
professionnels qu'il tente également de rapporter à des espaces sociaux plus larges et plus variés que l'organisation professionnelle. La conflictualité dans chacun de ces espaces joue chez lui un rôle considérable et l'on pressent qu'Anne-Chantal Hardy serait portée à nuancer, voire à contester ce postulat, elle qui souligne les sujétions et les allégeances auxquelles la profession doit son institutionnalisation. De même, Abbott s'attache à l'abstraction des savoirs qu'il considère comme la "monnaie ultime de la concurrence entre les professions » dans tous les espaces où la profession s'institue. Anne-Chantal Hardy la comprendrait plutôt dans le cadre d'un apprentissage forcé d'une autonomie qui sera ensuite contrôlée à distance par différentes institutions régulatrices. Pour Abbott cependant, ces savoirs abstraits offrent aussi une solution à l'obsolescence régulière des professionnels au fil de leur carrière. N'est-ce pas là une proposition intéressante pour les praticiens du soin? Bref, l'ouvrage d'Anne-Chantal Hardy fait espérer une suite, ce qui est bon signe.

Fermant l'ouvrage, on ne dispose donc pas d'une vision totalisante de la médecine, mais, certainement, d'une idée plus juste de cette profession. Son autonomie archétypale est vérifiée, mais uniquement pour la part de son activité qui la lie aux profanes, à ceux qui constituent sa matière et ses objets : les patients et leur corps. Pour les rapports avec les instances légitimantes, les sujétions et les allégeances fondées sur des coalitions de classes priment sur les luttes pour la reconnaissance. L'objet du travail médical, les notions de soin et de guérison ont perdu de leur évidence, mais on a compris l'urgence de s'y intéresser et d'éclairer, par le débat public, ce qui se fait aujourd'hui au nom de ce tourment qu'est, depuis le début de l'humanité, la guérison et qui nous concerne tous de si près.

\section{NOTES}

1. Parsons T. (1955), Éléments pour une sociologie de l'action, Paris, Plon, chap. 3 : «Structure sociale et processus dynamique. Le cas de la pratique médicale moderne ».

2. Freidson E. (1984), La profession médicale, Paris, Payot (1 $1^{\text {re }}$ éd. 1970).

3. Jamous H., Peloille B. (1970), "Professions or self-perpetuated systems? Changes in the French university-hospital system ", in Jackson J. A. (ed.), Professions and Professionalization, London, Cambridge University Press, pp. 109-152.

4. Abbott A. D. (1988), The system of professions: an essay on the division of expert labor, Chicago, University of Chicago Press, $452 \mathrm{p}$. 


\section{AUTEURS}

\section{SYLVIE CÉLÉRIER}

Centre d'études de l'emploi, Centre Pierre-Naville, Université d'Évry-val-d'Essonne 